\title{
Attempt to Transfer Contact Hypersensitivity in Man with Dialysate of Peripheral Leukocytes
}

\author{
Michael W. Brandriss \\ From the Department of Medicine, Rochester General Hospital and \\ the University of Rochester School of Medicine and Dentistry, \\ Rochester, New York 14621
}

A в S T R A C T In humans delayed hypersensitivity to tuberculin and coccidiodin can be transferred from a skin-test positive donor to a previously nonreactive recipient with a dialyzable fraction of donor peripheral leukocytes. The mechanism by which transfer is effected by this relatively low molecular weight material is unknown. Should transfer with dialysate be accomplished with hypersensitivity to low molecular weight artificial antigens, further characterization of "transfer factor" might be facilitated.

A maximal degree of contact sensitivity to a simple chemical, dinitrochlorobenzene, was produced in tuberculin-sensitive human volunteers, who were then used as donors. Seven of the eight donors reacted with $15 \mathrm{~mm}$ or more of induration to $0.1 \mu \mathrm{g}$ of tuberculin, purified protein derivative, while recipients did not react to $5 \mu \mathrm{g}$. The dialyzable fraction of peripheral leukocytes from $500 \mathrm{ml}$ of donor blood was injected into each of eight recipients in an attempt at simultaneous transfer of systemic sensitivity to both tuberculin and dinitrochlorobenzene. Transfer of tuberculin sensitivity was accomplished with four of seven dialysates from tuberculin-positive donors but in none of the eight attempts was contact sensitivity transferred with the same dialysates.

This paper was presented in part at the National Meeting of the American Federation for Clinical Research, Atlantic City, N. J., May 1968.

Dr. Brandriss' present address is the Rochester General Hospital, 1425 Portland Avenue, Rochester, N. Y. 14621.

Received for publication 21 February 1968.
The results indicate that tuberculin sensitivity and contact type sensitivity to a simple chemical differ in respect to the behavior of transfer factor and that other models are necessary if dialyzable transfer factor is to be studied with nonbacterial antigens.

\section{INTRODUCTION}

Lawrence and others have demonstrated, that in humans, the injection of an extract of peripheral leukocytes from a hypersensitive donor into a previously nonreactive individual can produce in the recipient a specific state of generalized delayedtype skin reactivity to tuberculin and to other bacterial or fungal products (1). The manner in which this transfer is effected and the factor ( $(\mathrm{s})$ responsible are unknown. "Transfer factor" can be dialyzed from disrupted donor leukocytes and the dialysate is as effective as the entire leukocyte extract in its ability to confer sensitivity to tuberculin and to coccidiodin $(2,3)$. The mol wt of the dialyzable factor is probably less than 40,000 and results of Sephadex G-25 fractionation have suggested a mol wt of less than 10,000 for the biologically active material (2).

Further characterization of transfer factor might be greatly facilitated if the transfer system could be applied to hypersensitivity to artificial, low molecular weight chemical allergens rather than to tuberculin or other complex naturally occurring bacterial and fungal products.

In the present experiments, contact sensitivity to dinitrochlorobenzene was induced in tuberculin- 
positive donors, and leukocyte dialysate from these individuals was injected into nonreactive recipients in an attempt at simultaneous transfer of both tuberculin and contact sensitivity. In this manner, a "positive control" of tuberculin sensitivity was introduced in the attempt to transfer contact sensitivity. Whereas transfer of tuberculin sensitivity was successful, transfer of contact sensitivity to dinitrochlorobenzene with the same dialysates was not achieved.

\section{METHODS}

Volunteers. Volunteers were adult male inmates of the Attica State Prison, Attica, N. Y. Subjects who were used as donors had no history of hepatitis, jaundice, blood transfusions, or drug addicition. Liver function tests (bilirubin, alkaline phosphatase, serum glutamic oxalacetic, and pyruvic transaminase) were normal. It was felt that the possibility of transmission of hepatitis virus was remote in any case since only the dialysate of disrupted leukocytes was injected into the recipients.

Production of contact sensitivity. 1-chloro-2,4-dinitrobenzene $^{1}$ (DNCB) was used. A suitable site on the outer aspect of the upper arm was scrubbed vigorously with $5 \%$ sodium lauryl sulfate, rinsed well with water, and dried. This left an erythematous area on which the DNCB was applied. On day $0,0.1 \mathrm{ml}$ of a $5 \%$ solution of $\mathrm{DNCB}$ in acetone, corresponding to a total dose of $5000 \mu \mathrm{g}$, was applied within a 1 inch diameter circle bounded by a glass ring tightly applied to the skin. The acetone was evaporated rapidly with a hair dryer leaving crystals of DNCB deposited on the skin. The site was covered with a Band-Aid for the next several days. On day 4, the same procedure was repeated at another site using $1000 \mu \mathrm{g}$ of DNCB.

Tests for contact sensitivity to DNCB. A similar procedure was used for testing except that the skin site was washed and rinsed very lightly and was not erythematous at the application site. The amount used for the test applications varied from 500-0.1 $\mu \mathrm{g}$. Test sites were examined at 24 and $48 \mathrm{hr}$, and usually at 3,4 , or 5 days.

Tuberculin testing. Tuberculin, purified protein derivative $^{2}$ (PPD) was used for all tuberculin tests. Volunteers were injected intradermally with $0.1 \mathrm{ml}$ of standard PPD solutions. Total doses were: first strength 0.02 $\mu \mathrm{g}$, intermediate strength $0.1 \mu \mathrm{g}$, and second strength 5.0 $\mu \mathrm{g}$ of PPD. Some donors were tested with more dilute solutions at total doses of $0.01,0.005$, and $0.001 \mu \mathrm{g}$ of PPD.

Preparation of dialysate of peripheral leukocytes. Peripheral leukocytes were processed after the procedure outlined by Lawrence (4). $500 \mathrm{ml}$ of blood was taken from hypersensitive donors in a Fenwal ion-exchange

1 Eastman Kodak Co., Rochester, N. Y.

2 Parke, Davis and Co., Detroit, Mich. blood pack. ${ }^{3}$ Fibrinogen, bovine fraction $1,40.05 \mathrm{~g} / \mathrm{ml}$ of blood, was added and the red cells were allowed to sediment for $1 \mathrm{hr}$ at $37^{\circ} \mathrm{C}$. The leukocyte containing supernate was then centrifuged at $1500 \mathrm{rpm}$ for $15 \mathrm{~min}$. The yield varied from approximately $1.5-2.5 \mathrm{ml}$ of packed leukocytes corresponding to approximately $1.4-2.5 \times 10^{\circ}$ cells. The sedimented cells were resuspended in a few milliliters of saline to which $0.1 \mathrm{ml}$ of $\mathrm{MgSO}_{4}, 1 \mathrm{~g} / \mathrm{ml}$, was added and quick frozen and thawed seven times. Beef pancreas deoxyribonuclease, ${ }^{5} 1 \mathrm{ml}$ of a $1 \mathrm{mg} / \mathrm{ml}$ solution, was added and the mixture was incubated in a $37^{\circ} \mathrm{C}$ water bath for $30 \mathrm{~min}$. The material was then placed in a dialysis sac and dialyzed against 100 volumes of pyrogen-free distilled water for $48 \mathrm{hr}$ at $4^{\circ} \mathrm{C}$. The dialysate was lyophilized and the product was redissolved in 2-3 $\mathrm{ml}$ of pyrogen-free distilled water.

The ultraviolet absorption spectrum of the dialysates was determined, and two dialysates were passed through a Sephadex G-25 column.

Recipients. Recipients were individuals who had negative reactions to $5 \mu \mathrm{g}$ of $\mathrm{PPD}$ on two occasions and who had had no known previous contact with DNCB. $1 / 10 \mathrm{ml}$ of dialysate was injected intradermally into the upper arm. This produced in most individuals an asymptomatic, erythematous reaction which appeared in $5 \mathrm{~min}$ and subsided over the next few hours. If there was no visible reaction at $24 \mathrm{hr}$ the remaining $1.9-2.9 \mathrm{ml}$ of dialysate was injected in divided doses intradermally and subcutaneously in the upper arm. Recipients were tested 24 or $48 \mathrm{hr}$ later with $5 \mu \mathrm{g}$ of PPD in the opposite forearm and with DNCB on the opposite upper arm.

\section{RESULTS}

Reaction to DNCB of control subjects. 51 applications of DNCB were made in 19 control subjects who had had no known previous contact with the chemical. Most subjects were tested simultaneously with 2 or 3 doses varying from 500 to $50 \mu \mathrm{g}$ of DNCB, and the results recorded here and in Table I refer only to these initial applications. Six of eight subjects developed erythematous, mildly edematous, nonpruritic reactions to $500 \mu \mathrm{g}$ of DNCB within $24 \mathrm{hr}$ of application. 5 of 12 developed similar but usually milder reactions to $250 \mu \mathrm{g}, 2$ of 19 developed very faint reactions to $100 \mu \mathrm{g}$, while none of 12 control subjects reacted to $50 \mu \mathrm{g}$ of $\mathrm{DNCB}$. These results are compared to those in actively sensitized subjects in Table I.

Active sensitization to DNCB. Contact hypersensitivity to DNCB was produced in all of 17 men in whom it was attempted. The sites of application of $5 \%$ DNCB became erythematous

\footnotetext{
${ }^{3}$ Fenwal Laboratories, Morton Grove, Ill.

4 Nutritional Biochemical Corp., Cleveland, Ohio.

5 Worthington Biochemical Co., Freehold, N. J.
} 
TABLE I

Skin Reactions to Application of $0.1 \mathrm{ml}$ of Dinitrochlorobenzene (DNCB) in Acetone in Actively Sensitized Subjects,

Controls (No Known Prior Contact with DNCB), and Recipients of Leukocyte Dialysate from

Hypersensitive Subjects

\begin{tabular}{cccc}
\hline $\begin{array}{c}\text { Total amount } \\
\text { DNCB applied }\end{array}$ & $\begin{array}{c}\text { Actively sensitized } \\
(17)^{*}\end{array}$ & $\begin{array}{c}\text { Controlsł } \\
(19)\end{array}$ & $\begin{array}{c}\text { Recipients } \\
(8)\end{array}$ \\
\hline$\mu g$ & & & \\
500 & - & $6 / 8 \S$ & - \\
250 & - & $5 / 12$ & $3 / 8 \S$ \\
100 & $17 / 17 \|$ & $2 / 19$ & $1 / 8$ \\
50 & - & $0 / 12$ & $0 / 8$ \\
20 & $17 / 17$ & - & - \\
10 & $15 / 17$ & & \\
5 & $11 / 17$ & & \\
1 & $8 / 17$ & & \\
0.5 & $3 / 17$ & & \\
0.1 & $0 / 17$ & & \\
\hline
\end{tabular}

* Total number in group.

$\ddagger 51$ applications of DNCB were made in the 19 controls. Most subjects were tested simultaneously with two or three concentrations.

\$ Numerator, number reacting to indicated concentration; denominator, number tested.

|| Numerator, number reacting to indicated concentration; denominator, total number in group.

with some induration and (or) brownish discoloration in $24-48 \mathrm{hr}$. In many subjects there was blistering and the epithelium loosened and peeled off. These reactions were not bothersome to the subjects except for a slight burning sensation in a few. The sites healed quickly and within 6-7 days were flat, showed only slight discoloration, and were asymptomatic.

Hypersensitivity became evident in 15 of the 17 subjects when one or both sites of the sensitizing applications flared into intensely red, elevated, itching reactions between day 8 and 11 . Test applications of DNCB (from 100 to $10 \mu \mathrm{g}$ ) were begun between day 10 and 14 and were continued, usually in decreasing concentrations, every week to 2 wk in most subjects. If an application resulted in a moderate to strong reaction a lower concentration was used next for a test dose. If the reaction was mild or negative, the same, or a more concentrated test dose was used. With this procedure, the degree of sensitivity increased in almost all subjects between 2 and 6 wk from the initial application of the sensitizing dose. Thereafter, there was a further increase in most subjects up to 10 wk after which no further increase in the degree of sensitivity was noted with repeated testing.

11 of the 17 subjects became sensitive to applications of $5 \mu \mathrm{g}$ or less of DNCB. 8 of these 11 subjects reacted to $1 \mu \mathrm{g}$ and 3 to $0.5 \mu \mathrm{g}$. No subjects reacted to less than $0.5 \mu \mathrm{g}$. Of the other 6 subjects, 2 who were tested repeatedly for 6 months never reacted to less than $20 \mu \mathrm{g}$ of DNCB. The other 4 subjects reacted to $10 \mu \mathrm{g}$ but were not available for a long enough period to determine what their ultimate degree of sensitivity might have been. The degree of reactivity of actively sensitized individuals is compared with that of control subjects in Table I. It was observed that even the mild reactions in actively sensitized subjects were almost always pruritic in contrast to the nonspecific reactions in control subjects which were asymptomatic.

Properties of leukocyte dialysate. The ultraviolet absorption spectrum of the dialysate was determined. All had an absorption peak at 252$255 \mathrm{~m} \mu$. A characteristic absorption spectrum is illustrated in Fig. 1. The shape of the curve is

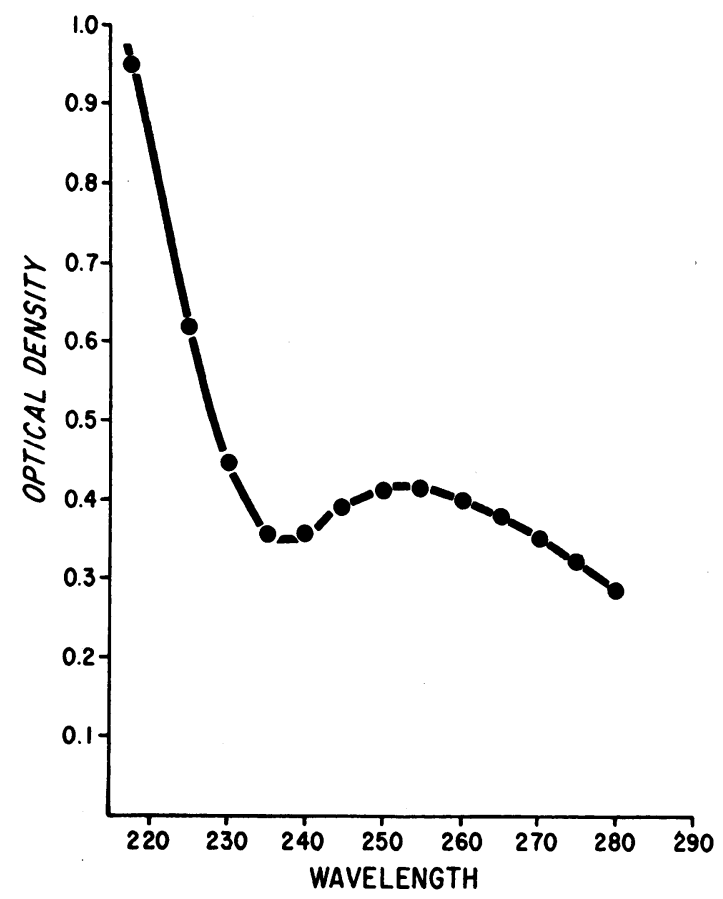

FIGURE 1 Ultraviolet absorption spectrum of leukocyte dialysate. 
similar to that found by Baram and Mosko although their peak was at $245 \mathrm{~m} \mu$ (3).

Two dialysates were passed through a Sephadex G-25 column after lyophilization and redissolution in a small volume of water. Two peaks were noted when OD at 260 and at $280 \mathrm{~m} \mu$ was plotted against the tube number of the eluate. This is illustrated in Fig. 2. The result is similar to that obtained by Lawrence, Al-Askari, David, Franklin, and Zweiman (2).

Attempts to transfer hypersensitivity to $P P D$ and $D N C B$. The degree of hypersensitivity to $\mathrm{PPD}$ and to $\mathrm{DNCB}$ of the donors is compared to that of the recipients in Table II. One recipient per donor was used in the transfer attempts. The eight subjects who were most sensitive to DNCB were used as donors. All reacted to applications of either 1 or $0.5 \mu \mathrm{g}$ of DNCB. Seven of the eight

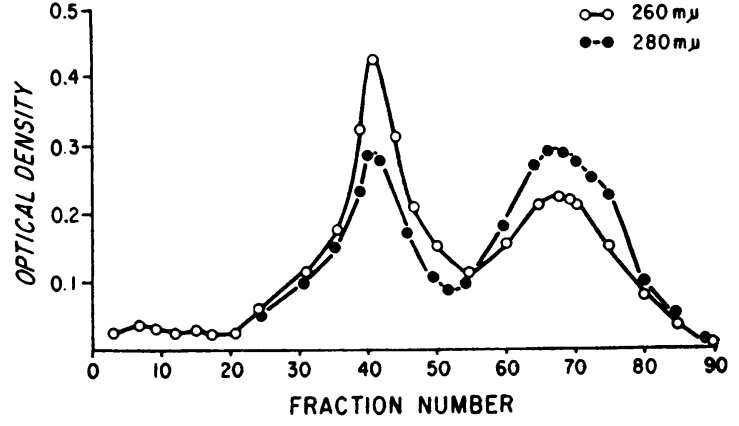

FIGURE 2 Leukocyte dialysate passed through Sephadex G-25 column; optical density vs. fraction number.

reacted with $17 \mathrm{~mm}$ or more of induration to injection of $0.1 \mu \mathrm{g}$ of PPD.

Four of the seven recipients of dialysate from PPD-sensitive donors developed delayed sensitivity to injection of $5 \mu \mathrm{g}$ of $\mathrm{PPD}$, but in no case

TABLE II

Reactivity of Donors and Recipients to Tuberculin, Purified Protein Derivative (PPD) and 2,4-Dinitrochlorobenzene (DNCB)

Donors and recipients are paired horizontally in the table.

\begin{tabular}{|c|c|c|c|c|c|c|}
\hline & \multirow{3}{*}{$\begin{array}{l}\text { PPD test } \\
\text { dose }\end{array}$} & \multicolumn{2}{|c|}{ Donors } & \multicolumn{3}{|c|}{ Recipients } \\
\hline & & \multirow{2}{*}{$\begin{array}{l}\text { Reaction } \\
\text { to PPD }\end{array}$} & \multirow{2}{*}{$\begin{array}{l}\text { Smallest dose } \\
\text { of DNCB to which } \\
\text { donor reacted }\end{array}$} & \multirow[b]{2}{*}{ Reaction to $5 \mu \mathrm{g}$ PPD } & \multicolumn{2}{|c|}{ Reaction to DNCB } \\
\hline & & & & & Test dose* & Result \\
\hline & $\mu g$ & $m m$ induration & $\mu g$ & $m m$ induration & $\mu g$ & \\
\hline 1. & $0.1 \ddagger$ & 32 & 1.0 & 12 & 250 & $0 \S$ \\
\hline 2. & $0.1 \ddagger$ & 25 & 0.5 & 0 & 250 & 0 \\
\hline 3. & $0.1 \ddagger$ & 4 & 1.0 & not done & 250 & 0 \\
\hline \multirow[t]{3}{*}{4.} & 0.1 & 17 & 1.0 & 0 & 250 & trace \\
\hline & 0.01 & 9 & & & 100 & 0 \\
\hline & 0.005 & 0 & & & & \\
\hline \multirow[t]{4}{*}{5.} & 0.1 & 17 & 1.0 & 25 & 250 & $+\|$ \\
\hline & 0.01 & 11 & & & 100 & 0 \\
\hline & 0.005 & 4 & & & & \\
\hline & 0.0025 & 0 & & & & \\
\hline \multirow[t]{4}{*}{6.} & 0.1 & 19 & 1.0 & 18 & 250 & 0 \\
\hline & 0.01 & 12 & & & & \\
\hline & 0.005 & 8 & & & & \\
\hline & 0.0025 & 0 & & & & \\
\hline \multirow[t]{5}{*}{7.} & 0.1 & 26 & 0.5 & 9 & 250 & + \\
\hline & 0.01 & 20 & & & 100 & trace \\
\hline & 0.005 & 17 & & & 50 & 0 \\
\hline & 0.0025 & 10 & & & & \\
\hline & 0.001 & 10 & & & & \\
\hline 8. & $0.1 \ddagger$ & 20 & 0.5 & 0 & 250 & 0 \\
\hline
\end{tabular}

* All recipients were tested with 250,100 , and $50 \mu \mathrm{g}$ of $\mathrm{DNCB}$. When there was no reaction to $250 \mu \mathrm{g}$ there was no reaction to smaller doses and these are not recorded.

$\ddagger$ Not tested with less than $0.1 \mu \mathrm{g}$ of PPD.

$\$ 0=$ no discernible reaction.

$\|+=$ weak but definite reaction. 
was reactivity to DNCB transferred. The reactions to $\mathrm{PPD}$ in the recipients varied from 9 to $25 \mathrm{~mm}$ in diameter of induration, were maximal at $24 \mathrm{hr}$, and were less prominent at $48 \mathrm{hr}$. Four of the seven donors were tested with dilutions of first strength PPD. Two donors reacted to $0.01 \mu \mathrm{g}$ but no less, one to $0.005 \mu \mathrm{g}$, and one to $0.001 \mu \mathrm{g}$ of PPD. (The $4 \mathrm{~mm}$ reaction of donor five to 0.005 $\mu \mathrm{g}$ of PPD was considered too small to be significant since it is difficult to evaluate a reaction of this size.) Dialysate from one of the two donors who reacted to $0.01 \mu \mathrm{g}$ failed to transfer reactivity to PPD. Successful transfers were accomplished with dialysate from the other three donors, although no correlation between the degree of donor and recipient reactivity was noted (Table II).

The recipients were tested with 250,100 , and $50 \mu \mathrm{g}$ of DNCB simultaneously at different sites. One of the eight recipients developed a trace of erythema at $100 \mu \mathrm{g}$ with no reaction at $50 \mu \mathrm{g}$. None of the other seven recipients reacted to $100 \mu \mathrm{g}$ of $\mathrm{DNCB}$ or less. Three of the eight recipients developed mild, erythematous, nonitching reactions to $250 \mu \mathrm{g}$ of DNCB (Table II). These reactions were similar to those seen in control subjects and the incidence of both are compared in Table I.

\section{DISCUSSION}

The characterization of transfer factor and means by which transfer of hypersensitivity is effected presents a challenging problem. Resistance to DNAse and RNAse as well as activity associated with material of probable mol wt of less than 10,000 (2) are not qualities expected of informational molecules that might instruct the production of an effector substance in the recipient. It is possible as Lawrence et al. have suggested (2) that dialyzable transfer factor could be a type of "micromessenger" RNA, a polynucleotide fragment below the level at which RNAse inactivation takes place. It is also possible that transfer factor is itself a relatively low molecular weight antibodylike effector material. Alternatively, it is possible that transfer factor contains antigen or antigenic fragments perhaps attached to nucleic acid fragments that result in active sensitization or a "boost" of a previously undetectable level of sensitivity in the recipient. Lawrence has demonstrated that there are two distinct temporal patterns of recipient response after transfer and has suggested that two different substances may play a role (4). Baram and Mosko have been able to transfer delayed hypersensitivity to tuberculin with both dialyzable and nondialyzable leukocyte fractions and with leukocyte fractions of differing chemical compositions $(3,5)$. These findings suggest that the transfer of delayed sensitivity in man need not be explained on the basis of a single mechanism of action of transfer factor(s).

Important advantages might be offered by a system utilizing leukocyte dialysate to transfer sensitivity to low molecular weight artificial antigens. One might, with more confidence, ascribe the reactivity in the recipient to a de novo sensitization rather than to a possible boost of a minimal underlying sensitivity to naturally occurring antigens. Isotopic labeling of low molecular weight chemicals might offer a practical means of determining whether antigen is present in transfer factor. Detection of possible in vitro reaction between antigen and transfer factor might be facilitated. In addition, the problem of the number of available donors and recipients would be alleviated. In the population used in this study, only $5-10 \%$ of individuals tested were suitable as recipients for tuberculin transfer. The practical difficulties presented by this can be appreciated if one considers the number of individuals necessary to establish a reliable model for transfer and, then, to vary the conditions of transfer.

Unfortunately, contact sensitivity to DNCB does not appear to be a useful model for this purpose. In this experiment many of the subjects received 15-20 applications of $\mathrm{DNCB}$ and the degree of donor sensitivity was taken to its practical limit with negative results.

There are several possibilities to explain the failure of the transfer of contact sensitivity with the simultaneous successful transfer of tuberculin sensitivity. First, contact sensitivity may represent something fundamentally different from intradermal delayed-type hypersensitivity that is not amenable to transfer with dialysate. Although there are reports of transfer of contact sensitivity with whole or disrupted cells (6-8), the results have been more erratic than those with transfer of intradermal type hypersensitivity (1). 
Alternatively, quantitative differences could have been significant in this experiment. In the case of the four donors who were tested with dilutions of PPD (Table II), there was at least a 500 -fold difference between the smallest test dose to which the donor responded $(0.01-0.001 \mu \mathrm{g})$ and the test dose used in the recipient $(5 \mu \mathrm{g})$. With donors four and five there was 500 -fold difference and one of those two transfer attempts with PPD was successful. (If the $4 \mathrm{~mm}$ reaction of donor five to $0.005 \mu \mathrm{g}$ of PPD were considered significant, the difference would then be 1000 -fold.) With donors six and seven, there was a 1000- and 5000 -fold difference, respectively, and both of these attempts were successful. In the case of DNCB (Table II) there was at most a 500-fold difference between the smallest dose to which the donor reacted $(0.5 \mu \mathrm{g})$ and the test dose used in the recipient $(250 \mu \mathrm{g})$. In two of these three transfer attempts (donors two, seven, and eight), the recipients failed to react to the $250 \mu \mathrm{g}$ test dose. The other five donors reacted to no less than 1 $\mu \mathrm{g}$ of $\mathrm{DNCB}$ and the difference between donor reactivity and recipient test dose was 250 -fold or less. It should be noted again that positive reactions to $250 \mu \mathrm{g}$ of $\mathrm{DNCB}$ in the recipients cannot be considered significant since some controls also reacted to this test dose (Table I). However, negative reactions can be considered significant in this context. The PPD system then, offered in most cases, a better opportunity to transfer in terms of the degree of donor sensitivity compared to the test dose that could. be used in the recipient, although the quantitative differences that were examined did not appear to be great. They could, nevertheless, be important.

Thirdly, one may be dealing with the difference between a true de novo immune response and a boost of an underlying state of minimal sensitivity.

The information available does not allow a choice between these alternatives. The question is being pursued at present with the use of haptenconjugated native human protein in an attempt to produce transferable delayed intradermal sensitivity.

\section{ACKNOWLEDGMENTS}

I wish to express my appreciation to the inmates of the Attica State Prison who participated in this study and to the warden and the administration for their help and cooperation.

This study was supported by U. S. Public Health Service grant AI 07352 from the National Institute of Allergy and Infectious Diseases.

\section{REFERENCES}

1. Lawrence, H. S. 1959. The transfer of hypersensitivity of the delayed type in man. In Cellular and Humoral Aspects of the Hypersensitive States. H. S. Lawrence, editor. Hoeber-Harper, New York. 279.

2. Lawrence, H. S., S. Al-Askari, J. David, E. C. Franklin, and B. Zweiman. 1963. Transfer of immunological information in humans with dialysates of leucocyte extracts. Trans. Assoc. Am. Physicians. 76: 84.

3. Baram, P., and M. M. Mosko. 1965. A dialysable fraction from tuberculin-sensitive human white blood cells capable of inducing tuberculin-delayed hypersensitivity in negative recipients. Immunology. 8: 461.

4. Lawrence, H. S. 1955. The transfer in humans of delayed skin sensitivity to streptococcal $M$ substance and to tuberculin with disrupted leucocytes. J. Clin. Invest. 34: 219.

5. Baram, P., L. Yuan, and M. M. Mosko. 1966. Studies on the transfer of human delayed-type hypersensitivity. I. Partial purification and characterization of two active components. J. Immunol. $97: 407$.

6. Epstein, W. L., and A. M. Kligman. 1957. Transfer of allergic contact-type delayed sensitivity in man. J. Invest. Dermatol. 28: 291.

7. Good, R. A., R. L. Varco, J. B. Aust, and S. J. Zak. Transplantation studies in patients with agammaglobulinemia. Ann. N. Y. Acad. Sci. $64: 882$.

8. Good, R. A., R. A. Bridges, S. J. Zak, and A. M. Pappenheimer, Jr. 1959. Delayed hypersensitivity in agammaglobulinemia. In Mechanisms of Hypersensitivity. Henry Ford Hospital International Symposium, Detroit, Mich., 1958. J. H. Shaffer, G. A. LoGrippo, and M. W. Chase, editors. Little, Brown and Co., Boston. 467. 